\title{
A CASE OF NARCOLEPSY.
}

BY JOHN MCGEE MACCORMAC, M.D.

Honorary Physician to the Victoria Hospital for Discases of the Nerrous System, Belfast.

As classical cases of narcolepsy are rare, I think the following one is of interest, more especially since a definite "exciting cause" seems to have existed.

The patient, Mary M., aged 27, came to the Victoria Hospital for Diseases of the Nerrous System, Belfast, on June 1, 1898, when the following history was obtained :-

Her parents and brothers and sisters are all healthy. She is a weaver by trade, and lived in the country until eleven years ago, and enjoyed good health up to about four years ago, when she had ten teeth extracted at one sitting without an anæsthetic. Immediately after this operation she commenced to feel unusually sleepy in the evenings, and soon afterwards began to sleep at her work, the sleep generally lasting from three to ten minutes at a time, and sometimes longer unless awakened. This sleep would overcome her under the most varied circumstances, and she found herself quite unable to prevent it. Between the attacks of sleep, which came on without warning, she felt quite clear, but complained of being more easily tired than natural. For some months after the extraction of the teeth she suffered from lightness in the head, with peculiar sensations passing over it from front to back, but these symptoms afterwards entirely disappeared.

On examination of the patient all the organs of the body were apparently healthy. She appeared mentally and physically lethargic, and walked slowly with a slight stoop. She had a heavy and rather sad expression, and her festures were somewhat puffy. She slept from seven to eight hours per night.

The following notes were afterwards taken :-

March, 1899.-The patient has steadily improved, especially during the last six months, the sleeping attacks having decreased 
from about twelve or more a day to one, and sometimes there is perfect freedom from them for one, two, or even three days. She looks brighter, and the expression is more natural.

May.-The patient has not attended the hospital for some weeks; and the sleeping attacks have become much more frequent again. Since the last notes the patient has lost her father, and has had other worries.

June.-Much improved again.

Treatment.-The medicines which seemed to act best were phosphate of iron, sulphate of quinine, tincture of nux vomica, and nitro-glycerine. She has had galvanism applied three or four times a week, a current of one milliampere being allowed to flow through the head from before back for three minutes at a time. She was ordered nutritious and easily digestible dietary, moderate exercise, and as much fresh air as possible.

I think we have here a typical case of narcolepsy, as evidenced by the persistency to fall asleep twelve or fourteen times daily, the ease with which the patient was awakened, and the complete consciousness during the intervals.

No symptoms pointing to hysteria or epilepsy were present, and the attacks of sleep resembled in all respects "natural sleep"; the face remaining unaltered in colour, the sleep lasting for several minutes and often longer, and the patient being quite easily awakened into complete consciousness. 